\title{
Deperdition Des Produits Sanguins Des Depots De Sang Aux Receveurs Dans Les Chu De Bouake Et De Treichville, Cote d'Ivoire
}

\author{
Kambé Kambé Yves, \\ Ouattara Kalilou, \\ Université Félix Houphouët-Boigny de Cocody, Abidjan, Côte d'Ivoire \\ Hyda Jules, \\ Centre National de Transfusion Sanguine (CNTS), Côte d'Ivoire \\ N'guessan Kouassi Bath Onésime,
}

Centre National de Transfusion Sanguine, President's Emergency Plan For

AIDS Relief (CNTS/PEPFAR)

Biérou Bodé Thomas,

Konaté Seidou,

Centre National de Transfusion Sanguine (CNTS), Côte d'Ivoire

Doi:10.19044/esj.2020.v16n15p309 URL:http://dx.doi.org/10.19044/esj.2020.v16n15p309

\section{Resume}

Cet article vise à évaluer la traçabilité des Produits Sanguins Labiles (PSL) dans les Centres Hospitaliers Universitaires (CHU) de Bouaké et de Treichville. L'étude est prospective et comparative. Elle a consisté à l'enrôlement exhaustif des bons de commande des Produits Sanguins Labiles (PSL) du 4 Août 2014 au 3 Octobre 2014, émis par les unités de soins issues des deux CHU susmentionnés et à suivre leurs destinations. Au total, 2152 unités de PSL ont été enrôlées et tracées. Cette évaluation révèle que pour 13 bons soit $0,6 \%$, le prescripteur de PSL n'existait pas dans le service de soins. Dans 166 cas sur les 2152 enrôlements réalisés, soit 7,7\% des cas, le patient mentionné sur le bon de commande n'était pas connu du service de soins et 424 soit $19,7 \%$ des PSL servis ne sont pas arrivés dans le service de soins, au lit du malade. Ces résultats sont faibles au CHU de Bouaké qui abrite un établissement de transfusion sanguine. En conclusion, la promiscuité ou la cohabitation Etablissements de soins-Etablissements de Transfusion Sanguine réduit la déperdition des Produits sanguins labiles.

Mots clés : Déperdition, Bouaké, Treichville, Produits Sanguins, Receveurs, Traçabilité 


\title{
Decrease of the Blood Component of the Hospital Blood Bank to the Receivers in Teaching Hospital Centers of Bouake and Treichville, Côte d'Ivoire
}

\author{
Kambé Kambé Yves, \\ Ouattara Kalilou, \\ Université Félix Houphouët-Boigny de Cocody, Abidjan, Côte d'Ivoire \\ Hyda Jules, \\ Centre National de Transfusion Sanguine (CNTS), Côte d'Ivoire \\ N'guessan Kouassi Bath Onésime, \\ Centre National de Transfusion Sanguine, President's Emergency Plan For \\ AIDS Relief (CNTS/PEPFAR) \\ Biérou Bodé Thomas, \\ Konaté Seidou, \\ Centre National de Transfusion Sanguine (CNTS), Côte d'Ivoire
}

\begin{abstract}
This article aims to evaluate the traceability of Labiles Sanguins Products (PSL) in the University Hospital Centres (CHU) in Bouaké and Treichville. The study is prospective and comparative. It consisted of the comprehensive enlistment of the order orders of The Sanguins Labiles Products (PSL) from August 4, 2014 to October 3, 2014, issued by the care units from the two aforementioned hospitals and to follow their destinations. A total of 2,152 PSL units were conscripted and traced. This evaluation reveals that for 13 vouchers or $0.6 \%$, the PSL prescriber did not exist in the care department. In 166 of the 2,152 enlistments made, or $7.7 \%$ of the cases, the patient mentioned on the purchase order was not known to the care department and 424 or $19.7 \%$ of the PSL served did not arrive in the care unit, in the patient's bed. These results are low at the Bouaké University Hospital, which houses a blood transfusion facility. In conclusion, the promiscuity or cohabitation Of Care Establishments-Blood Transfusion Establishments reduces the decrease of Labile Blood Products.
\end{abstract}

Keywords: Decrease, Bouaké, Treichville, Blood Products, Receivers, Traceability 


\section{Introduction}

Le don de sang en Côte d'Ivoire est basé sur le bénévolat, le volontariat et la non-rémunération. Conformément aux directives de l'OMS (1999), le Centre National de Transfusion Sanguine (CNTS) de Côte d'Ivoire est une structure publique qui a pour mission le prélèvement du sang pour la constitution de banques de sang, la qualification biologique des dons, l'approvisionnement des établissements de soins (ES) sur l'étendue du territoire ivoirien, la formation et la recherche. Le système transfusionnel ivoirien est centralisé (Bates et $a l, 2007$ ) et informatisé.

Depuis octobre 2004 jusqu'à ce jour, grâce à l'appui du PEPFAR et aux autres partenaires techniques et financiers, la transfusion sanguine en Côte d'Ivoire a connu un renforcement de ses services. Ainsi, beaucoup de réalisations ont été accomplies. D'abord il y'a eu la déconcentration des activités transfusionnelles. De de 04 structures en 2004, l'on en a comptait 27 en 2014. Puis, le développement d'un programme de recrutement et de fidélisation des donneurs à moindre risque pour le VIH s'est mis en place. Le nombre de poches de sang collecté s'est accru passant de 78.000 en 2004 à 143.691 en 2014. Le taux de diversification des produits sanguins est passé de $35 \%$ à $88,2 \%$ sur la même période. Enfin, le CNTS a mis en œuvre le renforcement des capacités des professionnels de santé des établissements de soins (ES) visant à améliorer l'usage clinique du sang (CNTS, 2015).

Le CNTS a une bonne maitrise des activités transfusionnelles de la collecte à la distribution et peut entreprendre au quotidien des actions pour pallier progressivement l'insuffisance en PSL de qualité. En effet, la cession des PSL se fait soit en lots (approvisionnement des dépôts de sang hospitaliers) soit de façon nominative (délivrance aux parents des malades). Cette opération est réalisée informatiquement et tracée dans la base de données du CNTS.

Cependant, une fois les produits sanguins distribués aux demandeurs, leur usage clinique dans les établissements sanitaires est méconnu du CNTS. Par ailleurs, des données non publiées du CNTS ont montré que certaines fois, les produits sanguins font l'objet de détournement au profit de bénéficiaires non identifiés ou non localisés, compromettant ainsi les efforts de traçabilité des unités de sang distribuées. Le présent article a eu pour but d'évaluer la déperdition des produits sanguins de la banque de sang au lit du malade

\section{Methodologie}

Notre travail est une étude prospective et comparative. Il s'est déroulé d'une part dans les Etablissements de Transfusion Sanguine (ETS) que sont l'Antenne de Transfusion Sanguine (ATS) de Bouaké et le Centre de Transfusion Sanguine (CTS) de Treichville et d'autre part dans les Etablissements de Soins (ES) à savoir les Centres Hospitaliers Universitaires (CHU) de Bouaké et de Treichville en Côte d'Ivoire. Le CHU de Bouaké 
abrite les locaux de l'ATS de la ville. A son tour, l'ATS abrite la banque de sang du CHU. C'est l'ATS qui gère donc toutes les activités de la chaine transfusionnelle c'est-à-dire du prélèvement à la distribution nominative du sang et des produits sanguins aux malades du CHU. Quant au CHU de Treichville, il n'abrite que la banque de sang. Le CTS de la commune qui l'approvisionne est situé à environ un kilomètre de ses locaux.

Quant à l'échantillonnage, au cours de l'étude, l'enrôlement exhaustif et concomitant de toutes les unités de PSL distribuées sur chaque site de l'étude, dans la période du 04 Août 2014 au 3 Octobre 2014 a été adopté.

Les données de l'étude ont été collectées à travers quatre sources à savoir le registre de distribution des produits sanguins labiles disponible au niveau des dépôts de sang hospitaliers, l'ordonnance médicale de PSL ou bon de commande, le logiciel médicotechnique du CNTS (Progesa) et la fiche journalière de renseignement.

Ainsi, trois méthodes ont été utilisées pour la collecte de données dans le cadre de cette étude à savoir la revue documentaire, l'extraction de données et l'observation. La revue documentaire a porté sur les registres de distribution des produits sanguins, les bons de commande de produits sanguins. La collecte de données a été faite à l'aide d'un questionnaire dénommé formulaire de collecte de données. L'extraction de données a concerné la base de données du CNTS hébergée par le logiciel médicotechnique Progesa. L'observation, quant à elle, a consisté à observer directement les poches de sang enquêtées dans les services de soins.

L'analyse desdites données a reposé sur la description des données démographiques des receveurs de produits sanguins. De même, les variables relatives aux prescriptions non-conformes, à la traçabilité des produits sanguins ont été décrites.

La saisie des données a été faite à partir d'un masque de saisie développé sous Epidata 3.1. L'analyse des données a été faite à l'aide du logiciel Stata 11.0.

\section{Considerations Ethiques}

Le protocole d'étude et autres documents afférents à savoir le formulaire de collecte de données, le formulaire de consentement ont été approuvés par le Comité National d'Ethique et de la Recherche de Côte d'Ivoire (CNER) et le Comité d'Ethique du CDC d'Atlanta (Institutional Review Board). 


\section{Resultats}

\subsection{Bon De Commande De Psl}

Tableau I : Bon de commande servis au cours de la période d'enquête

\begin{tabular}{|c|c|c|}
\hline Structure & Valeur Absolue & Valeur Relative \\
\hline CHU Bouaké & 1003 & $46,6 \%$ \\
\hline CHU Treichville & 1149 & $53,4 \%$ \\
\hline TOTAL & 2152 & $100,0 \%$ \\
\hline
\end{tabular}

Source : Notre enquête, 2014

Selon les données du Tableau I ci-dessus, 2152 Bons de commande de PSL ont été satisfaits dont 1149 soit 53,4\% au CHU de Treichville et 1003 soit 46,6\% au CHU de Bouaké.

\subsection{Traçabilité}

\subsubsection{Services de soins demandeurs de PSL dans les CHU}

Dans le cadre de la traçabilité des PSL honorés, la grande majorité des services mentionnés sur les bons de commande de sang (2 151 soit 99,95\%) existent et sont fonctionnels dans les structures hospitalières. Un seul cas d'inexistence et de non fonctionnement de service a été enregistré au CHU de Treichville (cf. tableau II).

Tableau II : Etat des services demandeurs de PSL au cours de la période d'enquête

\begin{tabular}{|c|c|c|c|c|}
\hline \multirow{2}{*}{ Structure } & \multicolumn{2}{|c|}{ Existence } & \multicolumn{2}{c|}{ Fonctionnalité } \\
\cline { 2 - 5 } & OUI & NON & OUI & NON \\
\hline \multirow{2}{*}{ CHU Bouaké } & 1003 & 0 & 1003 & 0 \\
& $100,00 \%$ & $0,0 \%$ & $100,00 \%$ & $0,0 \%$ \\
\hline CHU & 1148 & 1 & 1148 & 1 \\
Treichville & $99,95 \%$ & $0,05 \%$ & $99,95 \%$ & $0,05 \%$ \\
\hline \multirow{2}{*}{ TOTAL } & 2151 & 1 & 2151 & 1 \\
& $99,95 \%$ & $0,05 \%$ & $99,95 \%$ & $0,05 \%$ \\
\hline
\end{tabular}

Source : Notre enquête, 2014

\subsubsection{Déperdition des PSL des services de distribution au lit du malade}

Tableau III : Effectivité des médecins prescripteurs, patients et PSL au lit du malade

\begin{tabular}{|c|c|c|c|c|c|c|}
\hline \multirow{2}{*}{ Structure } & \multicolumn{2}{|c|}{$\begin{array}{c}\text { Médecin prescripteur } \\
\text { connu }\end{array}$} & \multicolumn{2}{c|}{$\begin{array}{c}\text { Patient mentionné sur le } \\
\text { bon de commande connu }\end{array}$} & $\begin{array}{c}\text { Arrivée effective dans le } \\
\text { service de soins du } \\
\text { malade }\end{array}$ \\
\cline { 2 - 7 } & OUI & NON & OUI & NON & OUI & NON \\
\hline CHU & 1002 & 1 & 982 & 21 & 950 & 53 \\
Bouaké & $99,9 \%$ & $0,1 \%$ & $97,9 \%$ & $2,1 \%$ & $94,7 \%$ & $5,3 \%$ \\
\hline CHU & 1137 & 12 & 1004 & 145 & 778 & 371 \\
Treichville & $99 \%$ & 1 & $87,4 \%$ & $12,6 \%$ & $67,6 \%$ & $32,4 \%$ \\
\hline \multirow{2}{*}{ TOTAL } & 2139 & 13 & 1986 & 166 & 1728 & 424 \\
& $99,4 \%$ & $0,6 \%$ & $92,3 \%$ & $7,7 \%$ & $80,3 \%$ & $19,7 \%$ \\
\hline
\end{tabular}

Source : Notre enquête, 2014 
La déperdition des PSL est constituée de trois variables : Il s'agit de l'existence du médecin prescripteur, de l'effectivité du patient mentionné sur le bon de commande et de l'arrivée du PSL au lit du malade. Les deux premières expliquent la déperdition. Quant à la dernière, elle traduit la fréquence du phénomène.

La variable "existence du médecin prescripteur" révèle que dans 13 cas $(0,6 \%)$, le médecin prescripteur dont l'identité figurait sur le bon de commande n'existait pas en réalité dans le service de soins où il était supposé être. Le CHU de Treichville représente la structure dont le taux de médecin prescripteur inconnu est le plus important avec 12 cas. Au niveau du CHU de Bouaké, ce constat est très faible avec 1 cas sur 1003.

Au niveau de l'effectivité du patient mentionné sur le bon de commande, dans 166 cas sur les 2152 enrôlements réalisés, soit 7,7\% des cas, le patient mentionné sur le bon de commande supposé être le bénéficiaire du PSL délivré n'était pas connu du service de soins où il était supposé être. Les données du CHU de Bouaké (21 soit 2,1\%) sont faibles au regard de celles du CHU de Treichville (145 soit $12,6 \%)$.

Quant à l'arrivée effective des PSL servis dans le service de soin, 424 cas de PSL servis soit $19,7 \%$ ne sont pas arrivés à destination c'est-à-dire au lit du malade. Le CHU de Treichville avec 371 sur $1146^{2}$ bons honorés soit $32,4 \%$ est la structure dont les taux de déperdition des PSL demeurent les plus élevés (cf. tableau III).

Les services les plus représentés sont les services d'urgences médicales et d'Accouchement/ Gynéco-Obstétrique pour le CHU Treichville avec respectivement 213 cas et 42 cas. Au niveau du CHU de Bouaké, le service Accouchement/ Gynéco-Obstétrique avec 21 cas est le plus représenté (cf. tableau IV).

D’une façon générale, les données du CHU de Bouaké demeurent faibles par rapport à celles du CHU de Treichville. La promiscuité voire la cohabitation ETS-ES) c'est-à-dire l'ATS et le CHU de Bouaké expliquerait-elle cette faible donnée de l'établissement de soin de ladite commune?

\footnotetext{
${ }^{2}$ Sur le total de 1149 bons honorés au CHU de Treichville, 3 bons ne portent aucune information sur le prescripteur. Ce faisant, l'analyse de cette partie porte sur les bons portant mention de l'identité du prescripteur.
} 
Tableau IV : Service croisé avec le nombre de cas où le PSL est non parvenu au lit du malade

\begin{tabular}{|c|c|c|}
\hline Services & CHU Bouaké & CHU Treichville \\
\hline Urgences Médicales & 5 & $\mathbf{2 1 3}$ \\
\hline Accouchement/ Gynéco-Obstétrique & 21 & 42 \\
\hline Urgences Chirurgicales & 10 & 39 \\
\hline Pédiatrie & 9 & 11 \\
\hline Chirurgie & 2 & 21 \\
\hline Médecine Interne & 2 & 28 \\
\hline Réanimation & 4 & 3 \\
\hline Cancérologie & - & 10 \\
\hline Maladies Infectieuses et Tropicales & - & 2 \\
\hline ORL & - & 1 \\
\hline Hémodialyse & - & $\mathbf{3 7 1}$ \\
\hline TOTAL & $\mathbf{5 3}$ & \\
\hline
\end{tabular}

Source : Notre enquête, 2014

\section{Discussion}

L'approvisionnement en sang de qualité et la compétence des professionnels de santé pour l'utilisation rationnelle du sang sont des variables essentielles pour le bon fonctionnement des services médicaux (Roberts et $a l$, 2016 ; CNTS, 2012).

Dans le cadre de la traçabilité des PSL, il existe des procédures strictes de gestion, d'attribution et si possible de réattribution des PSL non utilisés. Cependant la réattribution rencontre des difficultés dans les systèmes sanitaires des pays en développement en général et en Côte d'Ivoire en particulier. En effet, il n'existe aucune connexion (informatisation) entre les Etablissements de Soins (ES) et les Etablissements de Transfusion Sanguine (ETS).

Cette procédure a été décrite par Jeanne (2013) dans Gestion des transfusions loin d'un site de l'établissement français du sang. Il relève que l'éloignement d'un ES, des sites d'un ETS de l'établissement français du sang (EFS), impose différentes modalités de mise à disposition des PSL dans cet établissement, pour assurer une bonne gestion des transfusions des patients. Ainsi, les réattributions dans les dépôts ou services des ES quand des PSL délivrés par l'ETS référent ne sont pas transfusés au patient prévu, et que le temps requis est compatible avec la transfusion d'un autre patient, cette seconde délivrance est effectuée par l'ETS sans que le PSL ne quitte physiquement le dépôt ou le service. Elle fait l'objet d'une procédure établie entre l'ES et l'ETS, précisant les modalités de transmissions des données appropriées. De même, un PSL délivré par un dépôt peut être délivré une seconde fois, en respectant les principes des bonnes pratiques transfusionnelles. 
Par ailleurs, l'inadéquation évidente entre les besoins en produits sanguins labiles et les disponibilités dans les centres de transfusion sanguine engendre un drame quotidien, perceptible devant les maternités, les blocs opératoires et les services d'accueil des Urgences (Chobli, 2011). Ainsi, face à cette situation d'insuffisance de PSL pour satisfaire les besoins des patients voire de pénurie, les unités de soins préservent ou conservent les produits sanguins acquis des patients non transfusés de leur service pour d'éventuelle demande.

En outre, en cas de rupture de stock des banques de sang des établissements de soins, les parents de malades vont s'approvisionner directement dans les ETS qui sont le CTS de Treichville dans notre contexte. Notre étude a relevé un taux de déperdition de 19,7\% des PSL délivrés par les banques de sang hospitalières et le service de distribution du CTS de Treichville. Ces PSL ne sont pas arrivés pas au lit du malade. Nos données sont supérieures aux résultats du Burkina Faso où le taux de déperdition se situe entre $7,1 \%$ et $12 \%$ (Dahourou et al., 2012). La concordance entre le receveur programmé et le receveur effectif des produits sanguins était de 92,9 à 98,0 \% (Dahourou et al., op cit).

En outre, en Côte d'Ivoire, cette étude a permis de quantifier la déperdition des PSL et de fournir des données de traçabilité et d'hémovigilance qui demeure un maillon très faible de la sécurité transfusionnelle dans les pays en développement. Cette faiblesse est dépeinte par Ayob (2010). Il relatait que les données transfusionnelles ne sont que très peu renseignées mettant en mal les activités de traçabilité des produits sanguins labiles et d'hémovigilance et posant par ricochet la problématique des programmes nationaux d'hémovigilance dans les pays en développement. Ce constat est soutenu par la base de données de l'OMS 2004-2005 relative à la Sécurité du Sang qui indiquait que des systèmes nationaux d'hémovigilance sont présents dans seulement 42 des 105 pays (40\%). Pour sa part, Maotela (2015) souligne qu'il y a trop peu d'informations rapportées et documentées sur les indications de la transfusion sanguine, sur le déroulement de la transfusion ou les réactions per- et post-transfusionnelles.

Ainsi, réfléchissant sur la possibilité de la mise en place d'un système d'hémovigilance dans les pays d'Afrique subsaharienne, Dahourou et al. (idem) concluaient qu'elle était possible et qu'elle doit passer par une négociation entre les centres de transfusion et le personnel hospitalier encouragée par l'existence d'une réglementation officielle en matière de transfusion sanguine.

Par conséquent, la cohabitation de l'ATS et du CHU de Bouaké résout en grande partie ce problème. 


\section{Conclusion}

Cette étude a permis de quantifier le taux de déperdition des PSL du dépôt de sang au lit du malade. Il ressort que 19,7\% des PSL délivrés par les banques de sang hospitalières et le service de distribution du CTS de Treichville n'arrivent pas au lit du malade. La distance entre ES et ETS, et l'inadéquation évidente entre les besoins en PSL et les disponibilités dans les ES contribuent à cette déperdition. Ainsi, la cohabitation ES-ETS retenue par le CNTS dans sa politique de déconcentration de ses structures est une solution pour juguler le problème dans les ES partageant le même champ géographique. L'hémovigilance peut s'appuyer sur cette politique pour s'implanter à condition que les différentes parties s'y impliquent véritablement.

\section{Remerciements}

Les données ayant servi à la rédaction de cet article sont issues de l'étude sur la traçabilité des produits sanguins dans les quatre CHU de Côte d'Ivoire. Cette enquête a été réalisée grâce à l'appui du Plan d'Urgence du Président Américain pour la Lutte contre le VIH/SIDA (PEPFAR), à travers l'Accord de Coopération Numéro 1U2GPS002713 signé le 09 mars 2010 entre le Gouvernement Américain et le Ministère de la Santé et de la Lutte contre le Sida (MSLS) de Côte d'Ivoire.

Nous remercions ici tous les acteurs de cet organisme tant au niveau local qu'international.

\section{Conflit D'interet}

Aucun conflit d'intérêt.

\section{References :}

1. Ayob Y, 2010, Hemovigilance in developing countries, Biologicals, volume 38 numéro 1, pp. 91-6. doi: 10.1016/j.biologicals.2009.10.002. Epub 2010 Feb 4. Review.

2. Bates I, Manyasi G, Medina Lara A, 2007, Reducing replacement donors in Sub-Saharan Africa: challenges and affordability. Transfus Med., volume 17 numéro 6, p.434-42.

3. Bontez W, Bondurant A, 1993, Législation sur la transfusion sanguine en Côte d'Ivoire, Abidjan.

4. Centre National de Transfusion Sanguine (CNTS), 2012, Rapport d'activités 2011, Abidjan.

5. Centre National de Transfusion Sanguine (CNTS), 2015, Rapport d'activités 2014, Abidjan.

6. Chobli M, 2011, Quel sang pour quels patients en Afrique en 2011 ? Rev. Afr. Anesth. Med. Urg., Tome 16, n¹. 
7. Dahourou H, Tapkob J-B, Nebie Y, Kienou K, Sanou M, Diallo M, Barroa L, Murphyd E, Lefreree J-J, February 2012, Mise en place de l'hémovigilance en Afrique subsaharienne, Transfusion Clinique et Biologique, Volume 19, Issue 1, pp.39-45, http://dx.doi.org/10.1016/j.tracli.2011.11.001

8. Jeanne M, 2013, Gestion des transfusions loin d'un site de l'établissement français du sang, SFARVF3,http://sofia.medicalistes.org/spip/IMG/pdf/Gestion_des_tr ansfusions_loin_

d_un_site_de_1_etablissement_francais_du_sang.pdf

9. Kabinda Maotela J, Ramazani SY, Misingi P et Dramaix-Wilmet M, 2015, Transfusion sanguine en République démocratique du Congo : efforts réalisés et défis à relever, Médecine et Santé Tropicales, Volume 25, numéro 4, DOI : 10.1684/mst.2015.0487

10. Roberts Dj, Field S, Delaney M, Bates I, 2016, Problems and Approaches for Blood Transfusion in the Developing Countries, Hematol Oncol Clin North Am. ; volume 30 numéro 2, pp.477-95. doi: 10.1016/j.hoc.2015.11.011.

11. Organisation Mondiale de la Santé (OMS), 1999, Sécurité transfusionnelle, Aide-mémoire pour les programmes nationaux de transfusion sanguine, Genève. 